\title{
MÉTHODE DE DÉTERMINATION RAPIDE A L'USINE DES EXTRAITS SECS DES CONCENTRÉS
}

\author{
par \\ B. VIGOUREUX \\ Ingénieur Agronome
}

\section{I, Exposé de la méthode}

Cette méthode est basée sur Ia densité des constituants du concentré, que ces constituants soient à l'état dissous ou à l'état de suspension.

Cette densité que nous appellerons densité des constituants à l'état dissous ou suspendu, et qui peut différer de la densité usuelle des constituants à l'état sec ou isolé est le rapport exact du poids de ces constituants au volume qu'ils occupent dans la solution considérée.

Si nous insistons sur cette définition, c'est qu'elle est particulièrement nécessaire :

1. Dans le cas où l'on voudrait généraliser la méthode à d'autres produits concentrés que le lait (sirops, solutions concentrées de sels minéraux, solutions concentrées d'extraits de légumes ou de fruits, malts concentrés).

2. Dans le cas où l'on voudrait généraliser la méthode à d'autres solvants que l'eau ; on imagine en effet que la densité à l'état soluble d'une substance donnée puisse varier d'un solvant à l'autre en raison d'une affinité particulière et de la contraction moléculaire qui en résulte.

Dans le cas du lait qui nous intéresse particulièrement, ces densités ne sont autres que celles qui ont déjà fait l'objet de nombreuses études dont les dernières en date et les plus précises sont celles de Monsieur Jean Pren (relation de densités dans les produits laitiers : Revue Le Lait, juin 1938). C'est à ses chiffres que nous nous arrêtons :

Soit pour la matière grasse : 0,915 à $15^{\circ}$; et pour l'extrait dégraissé : 1,600 à la même température.

Mais à ces constituants qui définissent à eux seuls la totalité de l'extrait sec du lait, s'ajoute le saccharose qui est un constituant constant des laits concentrés.

Il nous a done fallu déterminer la densité du saccharose à l'état dissous. Cette densité nous a été fournie, par un calcul simple, sur les tables de LE BRIX, qui donnent le degré saccharimétrique des sirops en fonction de leur densité à $15^{\circ}$; elle est de 1,600 , ehiffre qui 
permet de confondre dans les calculs i'extrait dégraissé du lait et le sucre ajouté.

En bref, nous avons dans les laits concentrés trois constituants dont les densités respectives à $15^{\circ}$ sont :

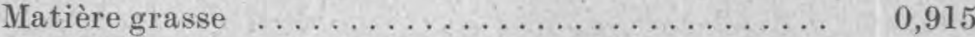

Extrait dégraissé $\ldots \ldots \ldots \ldots \ldots \ldots \ldots \ldots \ldots \ldots \ldots, 1,600$

Saccharose $\ldots \ldots \ldots \ldots \ldots \ldots \ldots \ldots \ldots \ldots \ldots, 1,600$

Ces densités nous permettent :

1. De ealculer la densité moyenne à $15^{\circ}$ des constituants de l'extrait sec.

2. Connaissant cette densité moyenne de calculer l'extrait sec pour 100 du concentré, selon sa densité à $15^{\circ}$.

\section{Calcul de la densité moyenne des constituants de l'extrait sec}

Si nous envisageons le cas général de $n$ constituants de densités respectives $d_{1} d_{2} d_{3} \ldots \ldots d_{n}$ et dont les proportions respectives en poids sont $\mathrm{a} \mathrm{b} \mathrm{c} \ldots \ldots \mathrm{n}$ la densité moyenne des constituants du mélange sera donnée par la formule :

$$
\frac{a+b+c+\cdots \cdots+n}{\frac{a}{d_{1}}+\frac{b}{d_{2}}+\frac{c}{d_{3}}+\cdots+\frac{n}{d_{n}}}
$$

fraction dont le numérateur représente le poids total d'extrait dans un volume quelconque de la solution et le dénominateur la somme des volumes occupés par chacun des constituants.

Cette formule qui figure dans l'étude très approfondie de Hunziker (Milk and Milk powders) n'est que l'application directe de la formule :

$$
\frac{\mathrm{P}}{\mathrm{V}}=\mathrm{D}
$$

Supposons done un lait partiellement écrémé contenant 116 grammes d'extrait sec par litre, dont 25 grammes de matières grasses. Avant la concentration, nous lui ajoutons par litre 65 grammes de saccharose.

La formule précédente nous donne la densité moyenne des constituants de ce lait :

$$
\mathrm{D}=\frac{25+91+65}{\frac{25}{0,915}+\frac{91}{1,600}+\frac{65}{1,600}}=1,450
$$


calcul simple qui n'exige, on le voit, que la connaissance de l'extrait sec et de la matière grasse du lait, éléments fournis immédiatement, si l'on fait usage de la nouvelle règle de Monsieur Jean PiEs, par une prise de densité et un Gerber.

Ce caleul, du reste, est évité par l'emploi d'un abaque.

\section{Calcul de l'extrait du concentré d'après sa densité à $15^{\circ}$}

Supposons que la densité du concentré obtenu soit de 1220 à $15^{\circ}$.

Nous sommes en droit de considérer ce concentré comme le mélange de deux constituants dont l'un aurait une densité de 1.450 et l'autre, l'eau, une densité de 1,000 (exactement : 0,999 à $15^{\circ}$ ).

Si nous nous rapportons au kilogramme de concentré, nous pouvons en utilisant la formule précédente, écrire :

$$
\frac{100}{\frac{x}{1,45}+\frac{100-x}{1}}=1,220
$$

dans laquelle $\mathrm{x}$ représente la teneur pour cent en extrait sec et 100 - $x$ le complément en eau.

$$
x=\frac{145 \times 0,22}{0,45 \times 1,22}=58,1 \%
$$

Cet exemple numérique n'a pour but que de rendre plus concrète l'étude ; nous allons établir maintenant les formules générales représentatives de ces applications.

Soit $\mathrm{d}$ la densité du concentré à $15^{\circ}, \mathrm{D}$ la densité moyenne des constituants à cette température et $\mathrm{x}$ la teneur pour cent en extrait sec ; nous obtenons les relations générales suivantes :

$$
\begin{aligned}
& d=\frac{100}{\frac{x}{D}+\frac{100-x}{I}} \\
& d=\frac{100 D}{x+(100-x) D} \\
& x=\frac{100 D(d-1)}{(D-1) d}
\end{aligned}
$$

Cette dernière formule nous permet de déterminer à tout moment de la concentration d'une liqueur quelconque sa teneur en extrait sec.

Ces ealculs ont été effectués pour toutes les densités de constituants prises de 10 en 10 de 1.250 (inclus) à 1.600 et pour les densités 
usuelles de concentrés (de 1.150 à 1.300 ), soit 800 résultats que nous avons regroupés dans un tableau à double entrée.

Nous ne le reproduisons pas ici puisque l'abaque dont nous allons maintenant parler y supplée.

\section{Construction et mode d'emploi de l'abaque}

L'abaque est constitué par une échèlle $A B$ graduée de 0 à 100 figurant les extraits secs et de deux échelles densimétriques semblables (voir abaque). L'une s'appelle échelle de densité des cons. tituants. Le point $\mathrm{G}$ y représente la densité de la matière grasse. L'autre s'appelle échelle de densité des concentrés. Le point C y représente la densité de l'eau.

Sur cet abaque peut s'effectuer :

10 Le calcul de la densité moyenne des constituants ;

$2^{\circ}$ Le calcul des extraits secs des concentrés.

a) Calcul de la densité moyenne des constituants.

Il faut d'abord convertir en \% de l'extrait sec le taux de matières grasses du lait sucré. Pour l'exemple précédemment choisi (lait à 25 grammes de matières grasses, 91 grammes de dégraissé et 65 grammes de saccharose) eette teneur est de $\frac{25}{181}=13,8 \%$.

Puis, à l'aide d'une équerre dont l'un des côtés de l'angle droit passe par la graduation 13,8 de l'échelle $\mathrm{AB}$ et dont le sommet $\mathrm{S}$ s'appuie sur la réglette $A G$, on lit en $D$ la densité moyenne des constituants. (Voir sur la figure I la position exacte de l'équerre.)

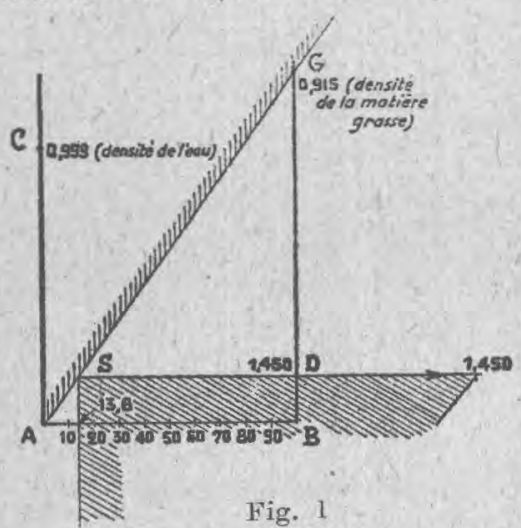

Recherche de D de l'extrait sec.

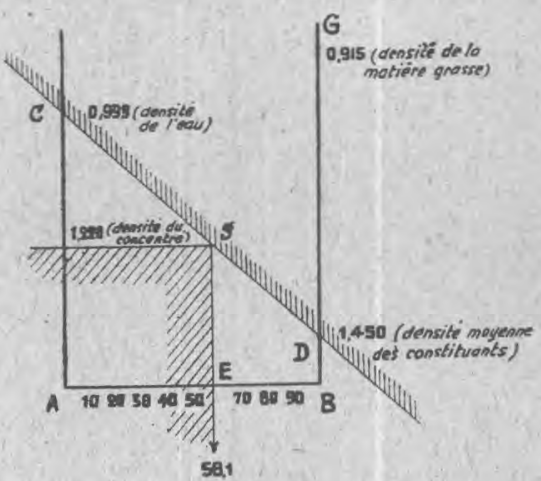

Fig. 2

Recherche de l'extrait.

b) Calcul des extraits secs des concentrés.

On place la réglette suivant CD et à l'aide de l'équerre dont l'un des côtés de l'angle droit passe par la densité du concentré à $15^{\circ}$ (soit 1.220 dans notre exemple), le sommet $S$ s'appuyant sur la réglette (voir figure 2) on lit en $\mathrm{E}$ l'extrait sec cherché. 


\section{Justification}

Soit $d_{1} d_{2} \ldots \ldots \ldots d_{n}$ les densités représentées par les points $\mathrm{E}_{1} \mathrm{E}_{2} \ldots \ldots \mathrm{E}_{\mathrm{n}}$ de la figure 3 . Les longueurs $\mathrm{OE}_{1} \mathrm{OE}_{2} \mathrm{OE_{n }}$ sont rigoureusement proportionnelles aux rapports $\frac{100}{\mathrm{~d}_{1}}, \frac{100}{\mathrm{~d}_{2}}, \frac{100}{\mathrm{~d}_{\mathrm{n}}}$.

A l'extrémité d'un segment tel que $\frac{100}{\mathrm{~d}}$ correspond donc la

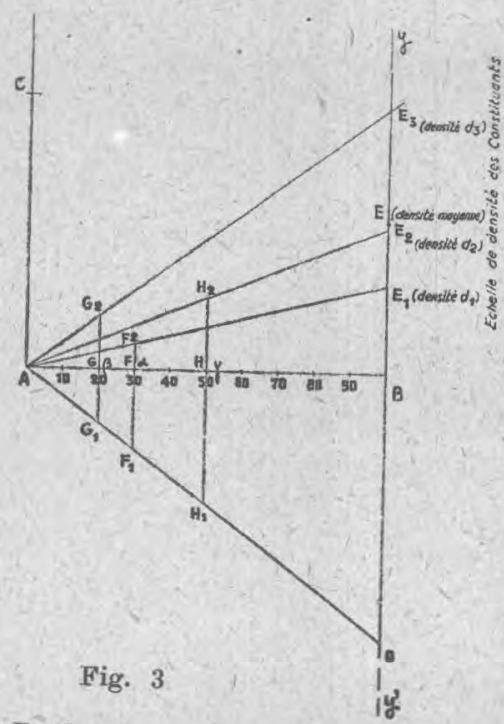

Recherche de la densité moyenne. dans le cas de 3 constituants. densité $d$.

a) Recherche de la densité moyenne (voir figure 3 ).

Supposons que l'extrait comprenne trois constituants dont les proportions relatives pour 100 grammes d'extrait soient $\alpha, \beta, \gamma(\alpha+\beta+\gamma=100)$.

Leurs densités respectives sont $d_{1}, d_{2}, d_{3}$ figurées par les points $\mathrm{E}_{1}, \mathrm{E}_{2}, \mathrm{E}_{3}$.

La densité moyenne sera :

$$
\Delta=\frac{100}{\frac{\alpha}{d_{1}}+\frac{\beta}{d_{2}}+\frac{\gamma}{d_{3}}}
$$

On observera que : $\frac{\alpha}{d_{1}}+\frac{\beta}{d_{2}}+\frac{\gamma}{d_{3}}=\frac{100}{\Delta}$.

Traçons $A E_{1}$ et par $F$ d'abscisse $\alpha$, eonstruisons le segment $F_{1} F_{2}$ perpendiculaire à $\mathrm{AB}$ et limité aux droites $\mathrm{AO}$ et $\mathrm{AE}_{1}$ ( $\mathrm{E}_{1}$ corres. pond à la densité de la substance en proportion $\alpha$ ).

Ce segment est égal aux $\frac{\alpha}{100}$ de $\mathrm{OE}_{1}$; puisque par construction : $\mathrm{OE}_{1}=\frac{100}{\mathrm{~d}_{1}}$

$$
\text { on a : } F_{1} F_{2}=\frac{\alpha}{100} \times \frac{100}{d_{1}}=\frac{\alpha}{d_{1}}
$$

de même : $G_{1} G_{2}=\frac{\beta}{d_{2}}$

$$
\mathrm{H}_{1} \mathrm{H}_{2}=\frac{\gamma}{\mathrm{d}_{3}}
$$


Additionner ces segments, c'est effectuer les calculs correspondants au dénominateur de la fraction (1).

$$
\begin{aligned}
& \text { Soit: } \frac{\alpha}{d_{1}}+\frac{\beta}{d_{2}}+\frac{\gamma}{d_{3}}=F_{1} F_{2}+G_{1} G_{2}+H_{1} H_{2}=1 \\
& \text { mais : } \frac{\alpha}{d_{1}}+\frac{\beta}{d_{2}}+\frac{\gamma}{d_{3}}=\frac{100}{\Delta} \\
& \text { donc } 1=\frac{100}{\Delta}
\end{aligned}
$$

D'après le principe de l'abaque, le point $\mathbf{E}$ obtenu en portant 1 sur $y^{\prime} y$ à partir de 0 porte la graduation $\Delta$ qui est la densité cherchée.

On observera que sont vérifiées les deux égalités suivantes :

$\mathrm{F}_{1} \mathrm{~F}_{2}+\mathrm{G}_{1} \mathrm{G}_{2}+\mathrm{H}_{1} \mathrm{H}_{2}=\mathrm{OE}$ (par construction)

$\mathrm{F}_{1} \mathrm{~F}+\mathrm{G}_{\mathbf{1}} \mathrm{G}+\mathrm{H}_{1} \mathrm{H}=\mathrm{OB}$ (puisque ces segments représentent respectivement les $\frac{\alpha}{100}, \frac{\beta}{100}, \frac{\gamma}{100}$ de $O B$ et que $\alpha+\beta+\gamma=100$.

Si nous soustrayons membre à membre, nous obtenons :

$$
\mathrm{FF}_{2}+\mathrm{GG}_{2}+\mathrm{HH}_{2}=\mathrm{BE}
$$

Ceci nous autorise à ne tenir aucun compte dans l'abaque de la partie située au-dessous de $\mathrm{AB}$ : pour obtenir $\mathrm{E}$, il nous suffit d'additionner les segments tels que $\mathrm{FF}_{2}, \mathrm{GG}_{2}, \mathrm{HH}_{2}$ et d'en reporter la somme à partir de $B$.

De là encore la grande simplification de l'abaque pour le lait concentré sucré : des 3 segments tels que $\mathrm{FF}_{2}, \mathrm{GG}_{2}, \mathrm{HH}^{2}$, deux sont rendus nuls par le choix du point $B: O B=\frac{100}{1,600}$; seul subsiste le troisième, directement reporté sur l'axe de densité par la première position de l'équerre.

b) Recherche de l'extrait (voir figure 4).

Soit $\Delta$ la densité de l'extrait : OE $=\frac{100}{\Delta}$

d la densité de l'eau : $O C^{\prime}=\frac{100}{d}$

$x$, la proportion d'extrait.

La démonstration est du même ordre que la précédente, les proportions des constituants sont $\mathrm{x}$ et $100-\mathrm{x}$ représentés par $J$ et $K$ et l'on observe que $J$ et $K$ sont symétriques l'un de l'autre par rapport à $\mathrm{M}$, milieu de $\mathrm{AB}$ 


$$
\mathrm{J}_{1} \mathrm{~J}_{2}+\mathrm{K}_{1} \mathrm{~K}_{2}=\mathrm{OE}^{\prime}
$$

on a comme précédemment :

$$
\mathrm{J} \mathrm{J}_{2}+\mathrm{K} \mathrm{K}_{2}=\mathrm{l}^{\prime}=\mathrm{BE}^{\prime}
$$

E', par sa position, détermine la densité d du mélange d'eau et d'extrait considéré, c'est-à-dire du concentré.

Soit C symétrique de C' par rapport à Z'Z. Considérons les droites $\mathrm{CE}$ et CB : ces droites coupent $\mathrm{JJ}_{2}$ en $J_{3}$ et $J_{4}$ ( $J$, nous l'avons $\mathbf{v u}$, est le symétrique de $\mathrm{K}$ et $\mathrm{J}_{4}$ le symétrique de $\mathrm{K}_{2}$ ).

Nous avons : $\mathrm{J} \mathrm{J}_{4}=\mathrm{K} \mathrm{K}_{2}$ par raison de symétrie ;

$$
\mathrm{J}_{3} \mathrm{~J}_{4}=\mathrm{J} \mathrm{J}_{2} \text { (appliquant le théorème de Thales, }
$$

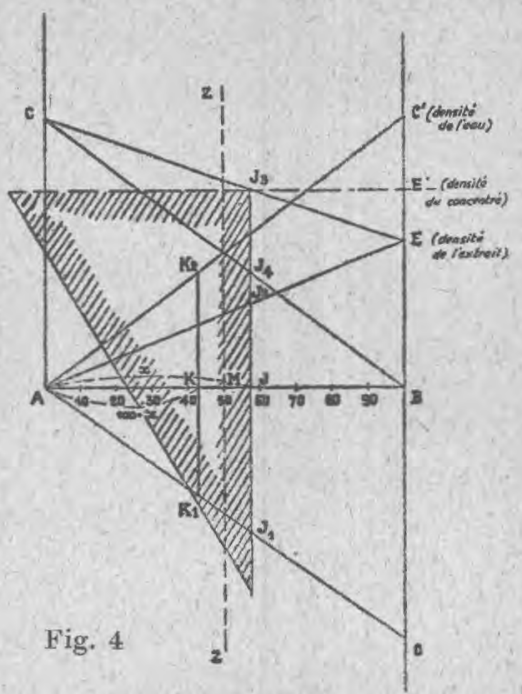

Justification géométrique de la recherche de l'extrait. aux triangles CEB et AEB, on observe que chacun de ces segments est égal aux $\frac{x}{100}$ d'un même segment $\mathrm{BE})$.

Done si nous additionnons membre à membre :

$$
\begin{aligned}
\mathrm{J} \mathrm{J}_{4}+\mathrm{J}_{3} \mathrm{~J}_{4} & =\mathrm{K} \mathrm{K}_{2}+\mathrm{JJ}_{2}= \\
\mathrm{l}^{\prime} & =\mathrm{J} \mathrm{J}_{3}
\end{aligned}
$$

La densité d, correspondant à l'extrait $\mathrm{x}$ nous est donnée par le segment $\mathrm{J} \mathrm{J}_{3}$ limité d'une part au point $J$ d'abscisse $x$; d'autre part au point $J_{3}$ sur CE ( $2^{\mathrm{e}}$ position de la règle) : ainsi se trouve justifiée la deuxième position de l'équerre.

\section{Critique de la méthode}

On voit que l'abaque traduit très fidèlement les formules proposées, sans introduire d'erreur et que la méthode est absolument générale.

Dans le cas du lait elle exige seulement le calcul du taux pour cent de matières grasses.

Il y a lieu toutefois de considérer que cette méthode est particulièrement sensible à la variation de ce taux, vu l'énorme écart entre la densité de cette substance et celle des autres constituants.

C'est ainsi que si la densité moyenne des constituants est de 1,450 pour un lait sucré contenant 25 grammes de matières grasses, 91 de dégraissé et 65 de saccharose, elle sera de 1,455 pour un lait 
contenant le même dégraissé et la même quantité de sucre mais seulement 24 grammes de matières grasses. Pour une densité de 1.220 l'extrait sec trouvé sera de 57,6 au lieu de 58,1.

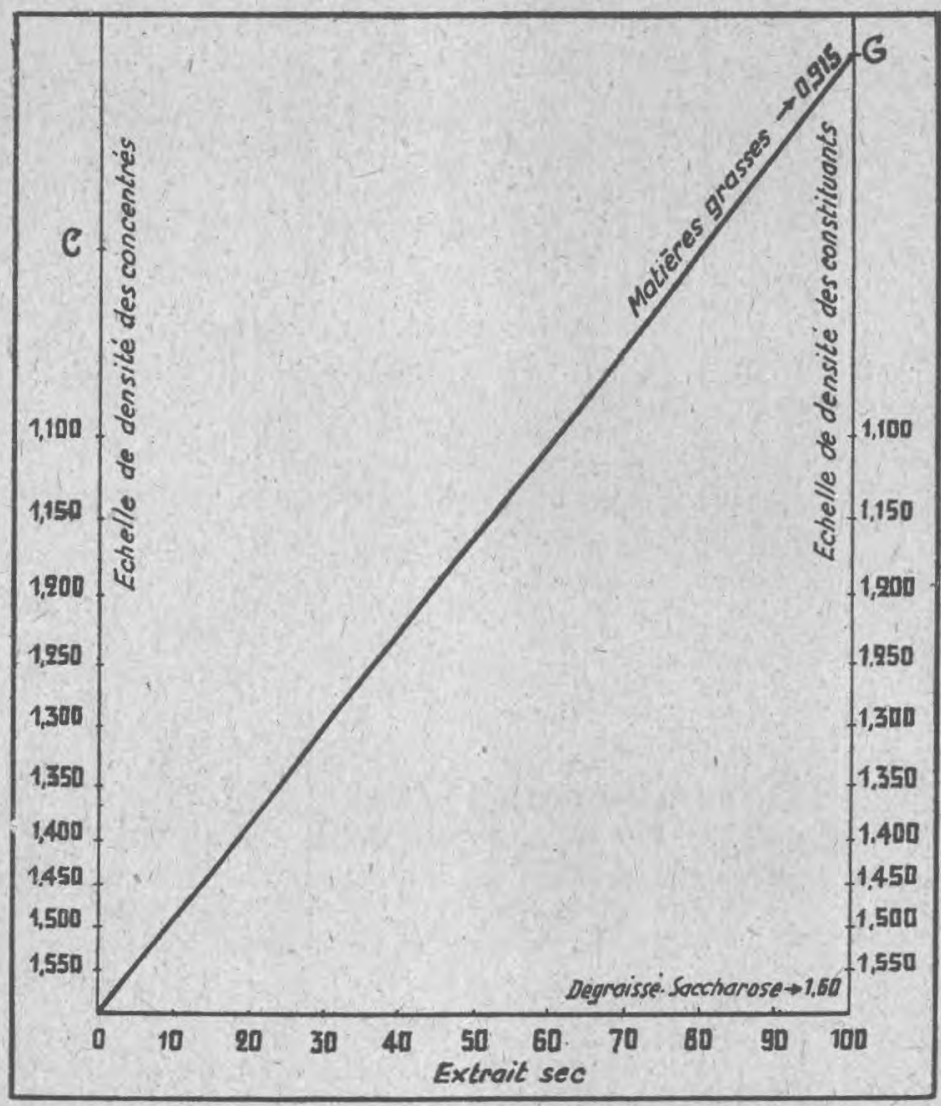

Fig. 5. - Abaque pour la détermination des extraits secs des. solutions ou suspensions concentrées (Réduction : 1/2).

Toutefois les erreurs commises sur la matière grasse au Gerber sont normalement faibles.

Cette remarque nous a pourtant autorisé à prendre $1,600 \mathrm{comme}$ densité du sucre, bien qu'aux concentrations usuelles cette densité apparaisse un peu supérieure. L'abaque et le ealcul en sont simplifiés et l'erreur commise sur le calcul de l'extrait sec n'est pas supérieure à celle de l'exemple précédent, ce qui fournit dans la pratique une suffisante précision.

Enfin, l'utilisation immédiate de l'abaque sur un lait en cours 
de concentration exige, si l'on n'a pas le moyen de refroidir rapidement ce lait, une correction de températ ${ }^{4}$ re, d'ailleurs variable avec la constifution du lait, sa teneur en sucre et sa concentration. (Nous aurons l'occasion de revenir sur cette question dans un prochain article.)

En résumé, la méthode fournit, dès maintenant, des résultats rapides et suffisamment exacts et de plus est susceptible de généralisation.

\title{
Note sur les méthodes volumétriques
}

Les méthodes volumétriques donnent une expression très simple de l'extrait sec en fonction de la densité, de l'extrait sec par litre du lait au départ de la concentration et en fonction de la densité du concentré.

Mais cette expression ne semble pas traduisible en abaque. D'autre part, le calcul de l'extrait sec par litre exigerait une nouvelle formule, du type Fleischmann, tenant compte du sucre ajouté et de la variation de volume due à cette addition. Son expression resterait complexe et les calculs deviendraient rapidement fastidieux. Enfin, cette formule se limiterait au cas particulier du lait et il faudrait en établir d'autres pour les autres prorluits.

\section{NOTES COMPLÉMENTAIRES RELATIVES A L'EMPLOI DU LACTOSÉRUM CONCENTRE DANS LAFABRICATION DES PATES ALIMENTAIRES (1)}

\author{
par \\ JEAN KIGER
}

Nous a vons antérieurement signalé ici même (2) les essais d'utilisation du lactosérum concentré dans la fabrication des pâtes alimentaires. Nous précisions alors les conditions d'emploi réalisées dans les essais auxquels nous a vions assisté et indiquions les caractéristiques analytiques des lactosérums employés, ainsi que des pâtes réalisées.

Depuis lors, nous avons suivi les nouvelles recherches effectuées, qui ont enfin abouti à la réalisation pratique de "pâtes au lactosérum " qui viennent d'être actuellement commereialisées dans la région de Dijon. Les lactosérums employés ont vu leur qualité très nettement améliorée et standardisée, de manière à donner des résultats de fabrication stables. Leur acidité, notamment, a été

(1) Bull. Soc. Sc. Hyg. alim., 1947, 35, 266.

(2) J. Kiger. - De l'emploi du lactosérum coneentré dans la fabrication des pâtes alimentaires. Bull. Soc. Sc. Hyg. alim., 1944, 32, 113. Le Lait, 1946, 26, 17. 